\title{
Escala de identidade para o exercício físico: validade fatorial e consistência interna para universitários brasileiros
}

https://doi.org/10.11606/issn.1981-4690.v35i1p35-42

\section{Resumo}

0 objetivo do presente estudo é verificar as propriedades psicométricas da Escala de Identidade para o Exercício Físico (EIS), no que diz respeito à validade de construto para o contexto brasileiro. Participaram do estudo 480 universitários dos cursos de Psicologia e Educação Física, dos estados de Santa Catarina, Minas Gerais e Brasilia, sendo 290 homens e 190 mulheres com média de idade de $23,7(d p=6,7)$ anos. Todos os 9 itens que compõem a escala mostraram carga fatorial adequada, justificando sua permanência na escala de acordo com os critérios previamente estabelecidos. A EIS validada cumpriu com as suposições de validade, com valores adequados de $\alpha$ de Cronbach e de correlações entre todos os itens, confirmando uma alta consistência interna da escala. Conclui-se que o EIS mostrou um bom desempenho psicométrico quando estudado a partir de uma amostra de universitários brasileiros.

Palavras-chave: Identidade; Atividade Física; Psicometria.

\section{Introdução}

Os benefícios do exercício físico, em termos de redução da mortalidade por todas as causas ${ }^{1,2} \mathrm{e}$ melhora dos marcadores de saúde mental ${ }^{3}$ estão bem estabelecidos na literatura ${ }^{4}$. No entanto, $62,1 \% \mathrm{da}$ população brasileira permanece insuficientemente ativa fisicamente ${ }^{5}$.

Nesse sentido, considerando a importância do exercício físico para a saúde pública, não é surpreendente que o apoio às pesquisas que verifiquem os fatores que moldam a decisão de iniciar, sustentar ou encerrar o envolvimento da prática física sejam desenvolvidos ${ }^{1,3,6}$.

Deste modo, um fator que pode auxiliar na aderência da prática de exercícios físicos é a identidade, estudos dentro da psicologia social e do esporte apontam a identidade como um fator fundamental na regulação de comportamentos dentro de diferentes estruturas sociais ${ }^{7-9}$. O desenvolvimento teórico desta linha de investigação baseia-se na interação entre a teoria da identidade social, decorrente do trabalho inicial de Tajfel e Turner ${ }^{10}$ e posteriormente na teoria da identidade ${ }^{11,12}$.

Deste modo, segundo Stets e Burke ${ }^{8}$ a identidade está relacionada com o grau em que o indivíduo está inserido (ou discriminado) de um grupo ou categoria social predefinido. Além disso, a teoria da identidade reconhece a importância das influências socioestruturais sobre a formação e o comportamento da identidade ${ }^{7,9}$.

As pesquisas na perspectiva da teoria da identidade concentraram-se predominantemente na importância dos papéis como fatores influentes que moldam a variabilidade comportamental durante a formaçáo da identidade ${ }^{8}$. A "identidade para o papel" representa uma posição social que é desenvolvida por meio de interaçóes com diferentes indivíduos em ambientes específicos ${ }^{11,13}$. As "identidades para o papel" representam conceitos atraentes para a compreensão dos comportamentos de persistência, dado que uma relação recíproca é postulada para existir entre a identidade para o 
papel e o investimento comportamental, no qual as pessoas irão regularizar seus comportamentos de uma forma consistente com os papéis que elas querem possuir ou adotar ${ }^{8,14}$.

Nesse sentido, estudos preliminares no domínio do exercício, demostram que quanto maior o grau que uma pessoa se identifica com o exercício físico praticado, maior será a frequência desse comportamento, bem como, a manutençáo futura desse hábito ${ }^{15-18}$.

Deste modo, um importante caminho para o avanço do estudo da identidade em contextos de exercícios físicos diz respeito ao desenvolvimento de instrumentos capazes de medir este construto. Um instrumento desenvolvido com o objetivo de medir como uma pessoa vê o exercício como parte integrante da sua autoidentidade é a Exercise

\section{Método}

\section{Tradução}

Para o processo de tradução e testagem empírica e analítica do instrumento foram realizadas as etapas metodológicas descritas nos tópicos abaixo. Aceitouse o construto teórico elaborado pelos autores concentrando-se na tradução e testagem empírica e analítica do instrumento em português.

A fim de garantir a qualidade da adaptaçáo do instrumento, este estudo seguiu os passos essenciais recomendados pela literatura especializada ${ }^{19-21}$. Primeiramente, a EIS foi traduzida por dois tradutores, obtendo-se assim duas versóes diferentes da escala: "T1 e T2". Após essa etapa, foi realizado um encontro com ambos os tradutores e o pesquisador principal, os quais definiram uma versão consensual "T1+2" em português.

A " $\mathrm{T} 1+2$ " foi entregue a um terceiro tradutor, que não conhecia o instrumento original. $\mathrm{O}$ questionário, "T1+2", foi traduzido para o inglês (retro-tradução). A versão original e a retrotradução foram comparadas e os pesquisadores estabeleceram uma versão consensual em português a "T3". Na qual, obteve-se a versão final traduzida para o português.

\section{Caracterização da pesquisa}

Trata-se de um estudo transversal, não probabilístico, com característica da amostra intencional e
Identity Scale (EIS) - Escala de Identidade para o Exercício Físico ${ }^{16}$. O desenvolvimento do EIS baseou-se principalmente em estudos anteriores de identidade para o papel, com intuito de avaliar o grau em que a participaçáo do exercício é importante no autoconceito de praticantes de exercícios físicos ${ }^{16}$, sendo validado para diferentes culturas avaliando trabalhadores e estudantes relacionados a área da saúde ${ }^{17,18}$. Assim, baseando-se em estudos prévios o EIS parece ser útil para o avanço da compreensão do construto identidade para o exercício físico. No entanto, nenhum estudo de validação referente a este instrumento foi encontrado no contexto brasileiro. Deste modo o objetivo do presente estudo é verificar as propriedades psicométricas da EIS, no que diz respeito à validade de construto, para o contexto brasileiro.

convencional ${ }^{22}$, de graduandos dos cursos relacionados à área da saúde, porém com diferenças quanto à suas práticas, em que a Educação Física como área prática do exercício físico, e a psicologia como uma área mais voltada à psique humana. $\mathrm{O}$ qual faz parte de um projeto maior intitulado "Identidade Esportiva e Artística de Atletas e Bailarinos", o qual foi desenvolvido pelo Laboratório de Gênero, Educação, Sexualidade e Corporeidade (LAGESC) da Universidade do Estado de Santa Catarina (UDESC). Projeto este submetido e aprovado pelo Comitê de Ética em Pesquisas com Seres Humanos (CEPSH) da UDESC sob o número de protocolo: 275.381/2013.

\section{Participantes}

Participaram do estudo 480 universitários dos cursos de Psicologia e Educação Física, dos estados de Santa Catarina, Minas Gerais e Brasília, sendo 290 homens e 190 mulheres com média de idade de 23,7( $d p=6,7)$ anos. Poderiam ser ou não praticantes de exercícios físicos, porém está variável não foi controlada para o presente estudo.

\section{Procedimentos de coleta dos dados}

Em um primeiro momento foi realizado um contato com as instituiçóes públicas e privadas que aceitaram participar do estudo e receberam a visita dos pesquisadores para esclarecimentos quanto 
à pesquisa frente aos responsáveis, apresentando o objetivo, relevância, procedimentos das coletas de dados, garantia do total sigilo de identificação na participação da pesquisa.

Os universitários que aceitaram participar assinaram o termo de consentimento livre e esclarecido e após recolhidos, as avaliaçóes foram realizadas. As coletas de dados ocorreram nas dependências das universidades e faculdades. A aplicaçáo dos questionários foi realizada em grupos, em salas de aula ou laboratórios, em que cada participante recebeu e respondeu os instrumentos de forma individual, recomendou-se que eles não conversassem durante o preenchimento dos instrumentos.

\section{Instrumento}

\section{Escala de Identidade do Exercício (EIS)}

Os participantes responderam a EIS composta por 9 itens desenvolvida por ANDERSON e Cychosz ${ }^{15}$ com o objetivo de avaliar a importância de se identificar com o exercício como parte integral do autoconceito. Os participantes responderam a cada item da EIS em uma escala ordinal de 7 pontos, com variaçáo de 1 discordo fortemente a 7 concordo fortemente. Pesquisas anteriores usando a EIS apoiaram validade e confiabilidade da escala ${ }^{18,22}$.

\section{Resultados}

\section{Validade de construto}

Os resultados obtidos carregaram em um único fator representando 66,2\% da variância. Recorreu-se ao teste de KMO, que apresentou valor de 0,953; o teste de Esferecidade de Bartlet $\left(x^{\wedge} 2(36)=5233,09\right.$ $\mathrm{p} \leq 0,001)$ e o $\alpha$ de Cronbach, igual a 0,936 para

\section{Análise dos dados}

\section{Escala de Identidade do Exercício (EIS)}

A validade do construto foi avaliada mediante a realizaçáo da análise fatorial exploratória com rotaçáo Oblimin, considerando um índice de carga fatorial de 0,3 para exclusão de itens ${ }^{23}$ e teste de Kaiser-MeyerOlkim (KMO) e esfericidade de Bartlett's para verificar a adequação da análise fatorial. Utilizou-se o gráfico Scree Plot para confirmar o número de fatores da escala. A consistência interna foi avaliada utilizando o teste Alpha de Cronbach e correlaçáo Policórica. Para estas análises os dados foram tabulados no programa estatístico Microsoft Excel $^{\oplus}$ e analisados no Statistical Package for the Social Sciences (SPSS) ${ }^{\circ}$ versão 20.

Fez-se o modelo de Análise Fatorial Confirmatória (AFC) por meio do programa estatístico STATA ${ }^{\circ}$ versão 13.1. Verificou-se a adequação dos modelos pelos seguintes parâmetros, Qui-quadrado $(\chi 2)$; Comparative Fit Index (CFI); Tucker Lewis Index (TLI); Root Mean Square Error of Approximation (RMSEA). Os valores de referência considerados para o Qui-quadrado $(\chi 2)$ foi um valor de $\mathrm{p}$ não significativo $^{24}$. O valor de 0,90 foi adotado como mínimo para inferir o ajuste do modelo, de acordo com os índices CFI e TLI ${ }^{24}$, enquanto para o RMSEA valores de zero a 0,08 como indicativo de ajuste aceitável ${ }^{24}$.

indicar a adequabilidade para a análise fatorial exploratória (TABELA 1).

O gráfico Screeplot confirma mais uma vez que o instrumento analisado carrega em um único fator considerando a dimensionalidade a partir de um ponto de corte de autovalor de 1,5 (FIGURA 1). 
TABELA 1 - Análise Fatorial Exploratória da EIS.

Itens

Carga fatorial

01 Eu considero-me um praticante de exercício

0,801

02 Quando eu me descrevo para os outros, eu usualmente incluo o meu envolvimento com o exercício

03 Eu tenho muitos objetivos relacionados a prática de exercícios

04 Exercício Físico é o fator central do meu autoconceito

05 Eu preciso praticar exercício para sentir bem comigo mesmo

06 Os outros me veem como alguém que pratica exercício regularmente

07 Para mim, ser um praticante de exercício significa mais que apenas me exercitar

0,805

08 Eu me sentiria realmente perdido se fosse forçado a desistir da prática de exercícios

\section{FIGURA 1 - Gráfico Screeplot da EIS}

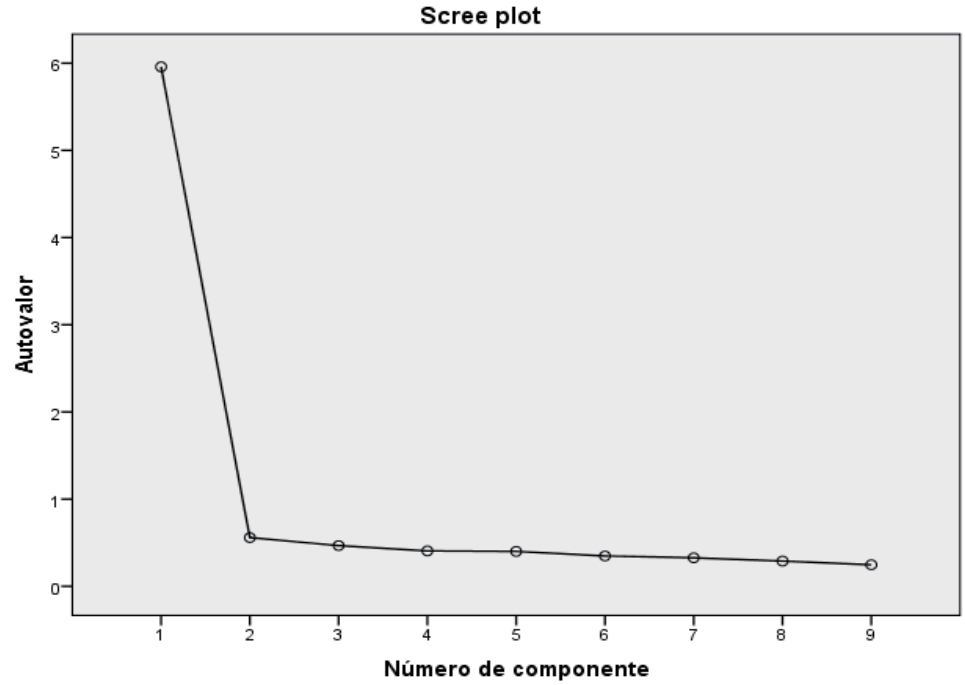

A consistência interna do EIS ainda foi testada por meio da correlação policórica entre os itens da escala. Conforme os dados apresentados na TABELA 2 todos os itens se correlacionaram significativamente ( $p>0.05$ ), confirmando uma boa consistência interna do instrumento (TABELA 2). 
TABELA 2 - Análise policórica dos itens da EIS.

\begin{tabular}{|c|c|c|c|c|c|c|c|c|c|}
\hline & I.1 & I.2 & I. 3 & I.4 & I. 5 & I.6 & I.7 & I.8 & I.9 \\
\hline I. 1 & 1,00 & & & & & & & & \\
\hline I. 2 & 0,75 & 1,00 & & & & & & & \\
\hline I. 3 & 0,69 & 0,75 & 1,00 & & & & & & \\
\hline I. 4 & 0,71 & 0,60 & 0,81 & 1,00 & & & & & \\
\hline I. 5 & 0,53 & 0,54 & 0,65 & 0,70 & 1,00 & & & & \\
\hline I.6 & 0,70 & 0,87 & 0,66 & 0,59 & 0,52 & 1,00 & & & \\
\hline I.7 & 0,79 & 0,65 & 0,72 & 0,74 & 0,63 & 0,80 & 1,00 & & \\
\hline I. 8 & 0,44 & 0,52 & 0,58 & 0,58 & 0,52 & 0,49 & 0,59 & 1,00 & \\
\hline I.9 & 0,70 & 0,67 & 0,75 & 0,73 & 0,61 & 0,75 & 0,90 & 0,58 & 1,00 \\
\hline
\end{tabular}

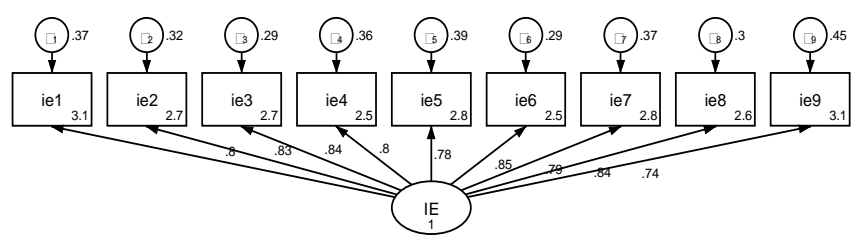

Ao analisar a estrutura fatorial do Modelo constituído por um fator da versão brasileira da EIS, verificou-se que o valor corrigido do $\chi 2$ não foi significativo $p>0.05$, o que sugere um forte ajuste dos seus itens, bem como, valores adequados para os índices de ajustamento: TLI
$(0,90)$, CFI $(0,91)$, índice da RMSEA $(0,045)$ e o índice SRMR $(0,041)$, confirmando a escala como unidimensional para o contexto investigado. A FIGURA 2 representa a estrutura graficamente confirmada da EIS adaptado para o contexto brasileiro. 


\section{Discussão}

Os resultados obtidos no processo de validação da EIS mostraram que os itens referentes à avaliação da identidade para o exercício físico possuem validade e confiabilidade suficientes para sua aplicaçáo em outros estudos sobre a temática no Brasil. Todos os 9 itens que compóem a escala possuem carga fatorial que justificam a sua permanência na escala de acordo com os critérios previamente estabelecidos. A EIS validada cumpriu com as suposiçóes de validade, com valores adequados de $\alpha$ de Cronbach e de $r$ entre todos os itens, confirmando uma alta consistência interna da escala.

Nesse sentido, Anderson e Cychosz ${ }^{15}$ forneceram evidência de validade estrutural em seu estudo inicial com estudantes universitários $(\mathrm{n}=51)$ usando análise fatorial exploratória foi encontrada solução de um fator para o construto, com uma variância total de explicaçáo de 67,6\%. Outras pesquisas de Anderson, Cychosz e Franke ${ }^{16,17}$ fornecem uma evidência adicional de validade da EIS, demostrando uma correlação positiva entre percepçôes mais fortes de identidade para o exercício e o comportamento fisicamente ativo ${ }^{16,17}$. Os efeitos relativos da idade também foram observados com pesquisas usando o EIS, segundo Anderson, Cychosz e Franke ${ }^{17}$ praticantes de exercício físico mais jovens apresentam maiores escores na EIS quando comparados a praticantes de exercícios mais velhos.

Em conjunto, os itens da EIS no presente estudo apresentam bons índices de consistência interna corroborando os achados de ANDERson, CyChOsZ e Franke $^{17}$ e Cardinal e Cardinal ${ }^{18}$ o quais encontram um $\alpha$ de Cronbach de 0,82 e 0,95, respectivamente, as evidências disponíveis sugerem que o EIS é um instrumento confiável para mensurar o construto de identidade para o exercício físico. Assim como proposto inicialmente este construto pode auxiliar a explicar o processo psicológico social de tornar-se ou ser um praticante de exercício, por ser a identidade para o papel desenvolvida e criada a partir das interações sociais, pessoas podem engajar- se em comportamentos que consideram importantes para estarem de acordo com a identidade para o papel, como a de praticantes de exercício físico ${ }^{17}$.

Apesar do apelo teórico e prático inerente a este estudo, uma série de limitaçóes devem ser reconhecidas para estudos futuros utilizando o EIS. Primeiro, este estudo empregou amostragem intencional o que restringe a validade externa de nossos dados. Também, este estudo baseou-se em um projeto não experimental, usando corte transversal para amostragem dos dados o que pode impactar os níveis de identidade para o exercício. Não controlou o envolvimento com a prática de exercícios físicos dos participantes.

Em conclusão, a versão em português do EIS na composição de 9 itens mostrou um bom desempenho psicométrico quando estudado a partir de uma amostra de brasileiros. O instrumento em questão apresentou atributos satisfatórios de consistência interna e validade de construto. Ressalta-se que no contexto brasileiro não se identificou outro instrumento específico que avalie características da EIS. Desta forma, a disponibilidade deste instrumento favorece o desenvolvimento de novos dados que podem melhorar a efetividade das intervençóes relacionadas ao exercício físico no Brasil. Além disso, destaca-se que mesmo a escala sendo desenvolvida para a população norte americana, os resultados encontrados com a aplicação da mesma confirmam a validade para brasileiros. Futuros estudos interessados na ampliaçáo do construto identidade para o exercício devem examinar o EIS em outras amostras (por exemplo, pessoas com deficiência, adultos mais velhos), podendo abarcar desenhos longitudinais para fornecer uma visão adicional sobre a natureza das relações temporais entre componentes de identidade para o exercício e frequência de realização de exercícios. Sugere-se ainda que em estudos futuros se investigue outras propriedades psicométricas da EIS, como validade de critério e poder de discriminação dos itens por meio da Teoria de Resposta ao Item (TRI). 


\begin{abstract}
Identity scale for physical exercise: factor validity and internal consistency for Brazilian university students

The objective of the present study is to verify the psychometric properties of the Exercise Identity Scale (EIS), with respect to construct validity for the Brazilian context. A total of 480 university students from the Psychology and Physical Education courses in the states of Santa Catarina, Minas Gerais and Brasilia participated in the study, with 290 men and 190 women with a mean age of 23.7 (SD $=6.7$ ) years. All 9 items that make up the scale showed adequate factorial load, justifying their permanence in the scale according to previously established criteria. The validated EIS met validity assumptions, with adequate values of Cronbach's and correlation among all items, confirming a high internal consistency of the scale. It is concluded that the EIS showed a good psychometric performance when studied from a sample of Brazilian university students.
\end{abstract}

KeYwords: Identity; Physical Activity; Psychometry.

\title{
Referências
}

1. Bouchard C, Blair SN, Haskell WL, Physical activity and health. Champaign, IL: Human Kinetics; 2006.

2. Nocon M, Hiemann T, Müller-Riemenschneider F, Thalau F, Roll S, Willich SN. Association of physical activity with all-cause and cardiovascular mortality: a systematic review and meta-analysis. Eur J Cardiovasc Prev Rehabil. 2008;15(3):239-46.

3. Biddle SJH, Fox KR, Boutcher S. Physical activity and psychological well-being. London, UK: Routledge; 2000.

4. Arem H, Moore SC, Patel A, Hartge P, Berrington de Gonzalez A, Visvanathan K, et al. Leisure time physical activity and mortality: a detailed pooled analysis of the dose-response relationship. JAMA Intern Med 2015;175:959-67.

5. Vigitel Brasil. Hábitos dos brasileiros impactam no crescimento da obesidade e aumenta prevalência de diabetes e hipertensão. Brasília; 2016.

6. Bauman AE, Sallis JF, Dzewaltowski DA, Owen N. Towards a better understanding of the influences on physical activity: the role of determinants, correlates, causal variables, mediators, moderators, confounders. Am J Prev Med. 2002; 23(8):5-14.

7. Cast AD, Burke PJ. A theory of self-esteem. Soc Forces. 2002;80(3):1041-1068.

8. Stets JE, Burke PJ. A sociological approach to self and identity. In: Leary MR, Tangney JP, editors. Handbook on self \& identity. New York: The Guillford Press. Cummings; 2003. p. 123-152.

9. Stryker S, Burke PJ. The past, present, and future of an identity theory. Soc Psychol Q. 2000;63(4):284-297.

10. Tajfel H, Turner JC. An integrative theory of intergroup conflict. Soc Psychol Intergr Relations. 1979;33(47):74.

11. Stryker S. Symbolic interactionism: a social structural version. Menio Park, CA: Benjamin Cummings; 1980.

12. Burke PJ, Reitzes DC. An identity theory approach to commitment. Soc Psychol Q. 1991;54(3):239-251.

13. McCall G, Simons J. Identities and Interactions. New York, NY: Free Press; 1978.

14. Baumeister RF, Schmeichel BJ, Vohs, KD. Self-regulation and the executive function of the self. In Leary MR, Tanguay JP, editors. Handbook of self and identity. New York, NY: Guilford Press; 2003. p.197-217.

15. Anderson DF, Cychosz CM. Development of an exercise identity scale. Percept Motor Ski.1994;78:747-751.

16. Anderson DF, Cychosz CM, Franke WD. Association of exercise identity with measures of exercise commitment and physiological indicators of fitness in a law enforcementcohort. J Sport Behav. 1998;21(3):233-241.

17. Anderson DF, Cychosz CM, Franke WD. Preliminary exercise identity scale (EIS) norms for three adult samples. J Sport Behav. 2001;24:1-9.

18. Cardinal BJ, Cardinal MK. Changes in exercise behaviour and exercise identity associated with a 14-week aerobic exercise class. J Sport Behav.1997;20(4):377-386.

19. Hutchinson $A D$, Wilson C. Improving nutrition and physical activity in the workplace: a meta-analysis of intervention studies. Health Promot Int. 2012;27(2):238-49.

20. Guillemin F, Bombardier C, Beaton D. Cross-cultural adaptation of health-related quality of life measures: Literature 
Silva WR, et al.

review and proposed guidelines. J Clin Epidemiol. 1993;46(12):1417-32.

21. Alexandre NMC, Guirardello EDB. Adaptación cultural de instrumentos utilizados en salud ocupacional. Rev Panam Salud Publica. 2002;11(2):109-11.

22. Gaya A. Ciências do movimento humano: Introdução à metodologia da pesquisa. Porto Alegre: Artmed; 2008.

23. Damásio BF. Uso da análise fatorial exploratória em psicologia. Avaliação Psicol. 2012;11(2):213-228.

24. Hu L, Bentler PM. Cut-off criteria for fit indexes in covariance structure analysis: Conventional criteria versus new alternatives. Struct Equ Model Multidiscip J. 1999;6:1-55.

ENDEREÇO

Walan Robert da Silva

Rua Pascoal Simone, 158

848080-350 - Florianópolis - SC - Brasil

Submetido: $12 / 04 / 2018$

Revisado: 07/08/2018

E-mail: walanrobert@hotmail.com

Aceito: 22/08/2018

42 • Rev Bras Educ Fís Esporte, (São Paulo) 2021J an-Mar;35(1):35-42 